\title{
Impact of diet on tooth erosion
}

\author{
Abstracted from
}

Salas MM, Nascimento GG, Vargas-Ferreira F, Tarquinio SB, Huysmans MC, Demarco FF.

Diet influenced tooth erosion prevalence in children and adolescents:

Results of a meta-analysis and meta-regression. J Dent 2015; 43: 865-875.

Address for correspondence: FF Demarco, Federal University of Pelotas, School of Dentistry,

Gonçalves Chaves, 457, Centro, zipcode 96015568, Pelotas-RS, Brazil. E-mail: ffdemarco@gmail.com

\section{Question: What is the influence of diet on tooth erosion?}

Data sources PubMed, Web of Science, Scopus, Science Direct, EBSHost, Scientific Electronic Library online (SciELO).

Study selection Population-based cross-sectional and longitudinal studies assessing tooth erosion and diet, conducted in children and adolescents between eight and 19 years reporting on the permanent dentition were considered.

Data extraction and synthesis Studies were selected independently by two reviewers and standard data items extracted. Study quality was assessed using the STROBE (Strengthening the Reporting of Observational studies in Epidemiology) statement and NewcastleOttawa Quality Assessment Scale (NOS). The pooled effect of dietary habits on tooth erosion occurrence was calculated using a fixed and a random model (OR and $95 \% \mathrm{Cl}$ ).

Results Thirteen studies involving a total of 16,661 children were included. Eleven of the studies were cross-sectional and two longitudinal. Dietary habits data were mainly obtained from brief dietary assessments (69.2\%) with food amount (weighed or estimated) and food frequency questionnaires used less commonly (15.4\%). Most dietary assessments were self-administered (84.6\%), assessed diet on a single occasion (61.5\%) and required recalls of a week or more days or usual behaviours (46.2\%). Meta-analyses were carried out for carbonated/soft drinks, sports drinks, milk-based drinks, yogurt, confectionery and snacks and acidic natural fruit drinks. Higher consumption of carbonated drinks or acid snacks/sweets and for acid fruit juices increased the odds for tooth erosion, while higher intake of milk and yogurt reduced the odds of erosion (see table). Conclusions The evidence indicated that some dietary habits (soft drinks, acidic snacks/sweets and acidic fruit juices) increased the odds for erosion occurrence, while milk or yogurt produced a protective effect. Methodological issues were shown to partly explain the heterogeneity of the data for some dietary products.

\begin{tabular}{l|l} 
& Odds ratio $(95 \% \mathbf{C I})$ \\
\hline Carbonated drinks & $1.60(1.29-1.99)$ \\
\hline Confectionery and snacks & $2.31(1.13-4.73)$ \\
\hline Milk & $0.96(0.91-0.99)$ \\
\hline Natural fruit juice & $1.20(0.02-1.42)$ \\
\hline Sports drinks & $2.13(0.95-4.77)$ \\
\hline Yoghurt & $0.77(0.64-0.91)$
\end{tabular}

\section{Commentary}

The levels of dental erosion defined as the irreversible loss of tooth tissue by chemical processes not involving bacteria are increasing in children and adolescents worldwide. While rates ranging between $7.2 \%$ to $95 \%$ have been reported in studies, a recent meta-analysis undertaken by the same group as this current review has estimated the overall prevalence to be $30.4 \%$ (95\%IC 23.8-37.0). ${ }^{1}$

A wide range of databases have been searched for this review and there were no restrictions on date or language. The included studies came from a wide range of countries; England, Brazil, The Netherlands, India, USA, China, Jordan, Libya, Sri Lanka and Sudan, with sample sizes ranging from 605 to 3812 . All but two of the included studies were cross-sectional, and one concern here is whether the information collected on diet at any particular point in time is reflective of diet over a longer period. The review authors themselves also question the potential of the various methods being used to gather the dietary data influencing findings. As most of the instruments also required participants to recall consumption there is also the issue of recall bias.

While the analysis demonstrates support for an association between the carbonated drinks, natural acidic fruit juices and confectionery and snacks and a higher risk of tooth erosion, and a reduced risk in those with high consumption of milk and yogurt, all the analyses demonstrate a high degree of heterogeneity. The authors also indicate that there may be the possibility of publication bias in particular related to carbonated drinks, as studies with negative associations were poorly published. Although the data suggest an association between these diet items and erosion there are limitations to the evidence and higher quality longitudinal studies with robust collection of diet data would be helpful in clarifying the position further. However, diet is not the only factor and another recent review by Hermont et al. ${ }^{2}$ suggests that patients with eating disorders had more risk of tooth erosion $(\mathrm{OR}=12.4,95 \% \mathrm{CI}=4.1-37.5)$ and those who also self-induced vomiting had a higher risk (OR=19.6, 95\%CI=5.6-68.8). While these odds ratios are considerably higher, the data are based on metaanalysis of case-controlled studies so should be viewed with caution.

Derek Richards Department of Public Health, NHS Forth Valley and Centre for Evidence-based Dentistry, DHSRU, University of Dundee, Scotland.

1. Salas MM, Nascimento GG, Huysmans MC, Demarco FF. Estimated prevalence of erosive tooth wear in permanent teeth of children and adolescents: an epidemiological systematic review and meta-regression analysis. J Dent 2015; 43: 42-50.

2. Hermont AP, Oliveira PA, Martins CC, Paiva SM, Pordeus IA, Auad SM. Tooth erosion and eating disorders: a systematic review and meta-analysis. PLoS One 2014; 9: e111123. doi:10.1371/journal.pone.0111123

Evidence-Based Dentistry (2016) 17, 40. doi:10.1038/sj.ebd.6401164 